\title{
Direction-of-Arrival Estimation for Coprime Array Using Compressive Sensing Based Array Interpolation
}

\author{
Aihua Liu, Qiang Yang, Xin Zhang, and Weibo Deng \\ Department of Electronic and Information Engineering, Harbin Institute of Technology, Harbin 150001, China \\ Correspondence should be addressed to Qiang Yang; yq@hit.edu.cn
}

Received 16 October 2016; Revised 6 January 2017; Accepted 12 January 2017; Published 16 February 2017

Academic Editor: Aboulnasr Hassanien

Copyright (C) 2017 Aihua Liu et al. This is an open access article distributed under the Creative Commons Attribution License, which permits unrestricted use, distribution, and reproduction in any medium, provided the original work is properly cited.

\begin{abstract}
A method of direction-of-arrival (DOA) estimation using array interpolation is proposed in this paper to increase the number of resolvable sources and improve the DOA estimation performance for coprime array configuration with holes in its virtual array. The virtual symmetric nonuniform linear array (VSNLA) of coprime array signal model is introduced, with the conventional MUSIC with spatial smoothing algorithm (SS-MUSIC) applied on the continuous lags in the VSNLA; the degrees of freedom (DoFs) for DOA estimation are obviously not fully exploited. To effectively utilize the extent of DoFs offered by the coarray configuration, a compressing sensing based array interpolation algorithm is proposed. The compressing sensing technique is used to obtain the coarse initial DOA estimation, and a modified iterative initial DOA estimation based interpolation algorithm (IMCA-AI) is then utilized to obtain the final DOA estimation, which maps the sample covariance matrix of the VSNLA to the covariance matrix of a filled virtual symmetric uniform linear array (VSULA) with the same aperture size. The proposed DOA estimation method can efficiently improve the DOA estimation performance. The numerical simulations are provided to demonstrate the effectiveness of the proposed method.
\end{abstract}

\section{Introduction}

Antenna arrays are usually used to perform spatial sampling of imping electromagnetic waves for improving detection performance of the source signal, and direction-of-arrival (DOA) estimation is a major application area of antenna arrays. It is well-known that the number of sources that can be resolved with an $N$ element uniform linear array (ULA) using conventional subspace-based DOA estimation method like MUSIC [1] is $N-1$. In order to get more degrees of freedom (DoFs) and hence resolve more sources than the actual number of physical sensors, minimum redundancy array (MRA) is proposed to maximize the number of continuous virtual sensors in the resulting difference coarray with given number of physical sensors. However, there are no closed form expressions for the array geometry or achievable DoFs for the MRA. To overcome this problem, the nested array [2] and the coprime array [3] are proposed with the exact expressions of the virtual sensor locations and the achievable DoFs. The nested array can resolve $O\left(N^{2}\right)$ sources with $N$ sensors. And coprime array [3] and the extended coprime array [4] can resolve $O(M N)$ sources with $M+N-1$ and $2 M+$ $N-1$ physical sensors, respectively. There are also two kinds of generalized coprime array configurations proposed in [5]. The virtual array of a coprime array is actually a symmetric linear array. And there are usually holes in the virtual array [6]. Therefore, the virtual array of a coprime array is a virtual symmetric nonuniform linear array (VSNLA) rather than a filled uniform linear array (ULA) when holes exist in the virtual array.

The increased DoFs provided by the coprime structure can be utilized to improve DOA estimation performance, and there are two major categories of representative DOA estimation techniques that have been proposed recently to utilize these increased DoFs for coprime arrays: one is compressive sensing (CS) technique, such as orthogonal matching pursuit (OMP) [7] and the least absolute shrinkage and selection operator (LASSO) [8]. The LASSO algorithm is considered in [9-11] for sparse signal recovery using coprime array. The main drawback of CS technique is that every source must fall on the predefined grid, off-grid sources can highly jeopardize the reconstruction performance. To 
overcome dictionary mismatch problem for CS technique, joint-OMP and joint LASSO algorithms are proposed in [12], and an classic off-grid DOA estimation method using sparse Bayesian inference is proposed in [13], which also explores the underlying structure between the sparse signal and the grid mismatch.

The other representative DOA estimation technique for coprime array is based on the well-known MUSIC algorithm called MUSIC algorithm with spatial smoothing (SS-MUSIC) [2], which uses spatial smoothing technique $[14,15]$ to build a suitable covariance matrix from the virtual sensor output before applying MUSIC spectrum estimation [2, 4]. However, spatial smoothing cannot be used directly when the virtual array is not a filled ULA, which means that only the continuous lags in the VSNLA can be utilized for the application of spatial smoothing [5]. When the number of continuous lags in the VSNLA is lower than that of the unique lags in the VSNLA, the DoFs offered by coprime array are only partly explored, which significantly reduces the DOA estimation precision and the number of resolvable sources. To fully explore the virtual array aperture of the VSNLA using SS-MUSIC, on the one hand, multiple frequencies are utilized to fill the missing elements in the VSNLA [16]. However, it requires that the reflections of sources have large bandwidth to cover all specific frequencies used for filling the holes, and the sources spectrums at all operational frequencies should be proportional, which may be difficult to meet in the practical application. On the other hand, array interpolation techniques are proposed to fill the holes in the VSNLA and the sparsity-based extrapolation technique [6] uses sparse reconstruction to extrapolate observations at the holes of the VSNLA, but the basis mismatch problem at the sparse reconstruction stage is not considered. The coprime coarray interpolation technique is proposed in [17] to fill the holes by using nuclear norm minimization, while it needs to solve a complex semidefinite programming problem.

In this paper, we consider single frequency operation to utilize all of the DoFs for DOA estimation in coprime array using preprocessing method like interpolation [18] as mentioned in [2]. However, conventional interpolation techniques [18-21] cause big interpolation errors over the entire field of view due to the holes in the VSNLA of coprime array. To reduce interpolation errors, the idea of initial DOA estimation based array interpolation is used in $[22,23]$ by specifying a union of small subsectors that cover only the source directions inside. To obtain the initial DOA estimation, two different special nonuniform linear array (NLA) structures, partially augmentable nonredundant array [24] and partly filled NLA, are considered in [22], and the iterative initial DOA estimate based array interpolation approach (IMCI-AI) is proposed to solve multipath problem for the partly filled NLA. Like [22], the IMCI-AI method is used in this paper to build up the rank of the observation matrix of the VSNLA of coprime array. However, the initial DOA estimation of signals is the prerequisite of this method; although the SS-MUSIC can be used to obtain the initial DOA estimation with the continuous lags in the VSNLA, it has the limitation that the number of sources resolvable should be less than half that of the continuous lags in the VSNLA.

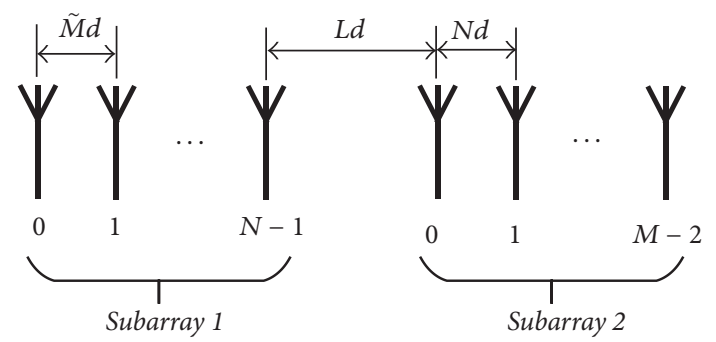

FIGURE 1: The coprime array with displaced subarrays (CADiS) [5].

To detect more sources and improve the DOA estimation accuracy for coprime array with VSNLA, a compressive sensing based array interpolation approach for DOA estimation is proposed in this paper. Firstly, the coarse initial DOA estimation of sources is obtained using the LASSO compressing sensing technique. Then, a modified IMCI-AI approach is used to interpolate the data of a symmetric uniform linear array (VSULA) using the received data of the VSNLA; after the interpolation, the holes in the VSNLA are filled, leading to a filled VSULA with the same array aperture as the VSNLA. Then, the ESPRIT-Like algorithm [25] is applied to the filled VSULA for the improved DOA estimation of sources using the symmetric structure of the VSULA. Compared with the VSNLA, the filled VSULA after interpolation has more continuous elements and it can be used to detect more sources. Like the traditional IMCI-AI method, the proposed interpolation approach can make full use of the array aperture offered by coprime array. However, the proposed method can resolve more sources. Compared with general CS algorithms, the proposed algorithm does not suffer from basis mismatch effects.

The remainder of this paper is organized as follows. In Section 2, the signal model of the VSNLA of coprime array is reviewed. In Section 3, DOA estimation using SS-MUSIC and the proposed array interpolation methods are compared. Simulation results are provided in Section 4. Finally, Section 5 concludes the study.

\section{Signal Model}

We consider generalized coprime arrays [5]; the coprime array with displaced subarrays (CADiS) in this paper is an example of coprime array with holes in its virtual array. The CADiS is illustrated in Figure 1. This kind of coprime array configuration consists of two collinearly located uniform linear subarrays: one has $N$ antennas with distance $\widetilde{M} d$ between two continuous antennas and the other has $M-1$ antennas with distance $N d$ between two continuous antennas, where $d$ is the unit spacing set to $\lambda / 2$, and $\lambda$ denotes the wavelength. $M$ and $N$ are coprime integers and $M$ can be expressed as a product of two positive integers $p$ and $\widetilde{M}(M=p \widetilde{M})$. The two subarrays are placed collinearly with the closest spacing between the two subarrays set to $L d$. The total number of the sensors in each coprime array is kept to $M+N-1$.

Denote $\mathbf{p}=\left[p_{1}, \ldots, p_{M+N-1}\right]^{T}$ as the sensor position vector of the coprime array, where $(\bullet)^{T}$ denotes transpose 
operator, and the first sensor is assumed as the reference, that is, $p_{1}=0$. Assume that $K$ far-field uncorrelated narrowband sources imping on the array from directions $\theta_{k}$, $k=1,2, \ldots, K$, and their discretized baseband waveforms are expressed as $s_{k}(t), k=1,2, \ldots, K$. Then, the received data vector at time $t$ is expressed as

$$
\mathbf{x}(t)=\sum_{k=1}^{K} \mathbf{a}\left(\theta_{k}\right) s_{k}(t)+\mathbf{n}(t)=\mathbf{A s}(t)+\mathbf{n}(t),
$$

for $t=1, \ldots, T$, and $\mathbf{A}=\left[\mathbf{a}\left(\theta_{1}\right), \ldots, \mathbf{a}\left(\theta_{K}\right)\right]$, where

$$
\begin{aligned}
& \mathbf{a}\left(\theta_{k}\right) \\
& \quad=\left[e^{j 2 \pi p_{1} \sin \left(\theta_{k}\right) / \lambda}, e^{j 2 \pi p_{2} \sin \left(\theta_{k}\right) / \lambda}, \ldots, e^{j 2 \pi p_{M+N-1} \sin \left(\theta_{k}\right) / \lambda}\right]^{T}
\end{aligned}
$$

is the manifold vector of the coarray corresponding to $\theta_{k}$, and $\mathbf{s}(t)=\left[s_{1}(t), \ldots, s_{K}(t)\right]^{T}$ is the signal vector, and $\mathbf{n}(t)$ is the noise vector supposed to be temporally and spatially white Gaussian which is uncorrelated with the sources. The covariance matrix of data vector $\mathbf{x}(t)$ is obtained as

$$
\begin{aligned}
\mathbf{R}_{\mathbf{x x}} & =E\left[\mathbf{x}(t) \mathbf{x}^{H}(t)\right]=\mathbf{A R}_{s} \mathbf{A}^{H}+\sigma_{n}^{2} \mathbf{I}_{M+N-1} \\
& =\sum_{k=1}^{K} \sigma_{k}^{2} \mathbf{a}\left(\theta_{k}\right) \mathbf{a}^{H}\left(\theta_{k}\right)+\sigma_{n}^{2} \mathbf{I}_{M+N-1},
\end{aligned}
$$

where $E(\bullet)$ is the statistical expectation operator and $\mathbf{I}_{M+N-1}$ is an $(M+N-1) \times(M+N-1)$ identity matrix. $\mathbf{R}_{s}=$ $E\left[\mathbf{s}(t) \mathbf{s}^{H}(t)\right]=\operatorname{diag}\left(\left[\sigma_{1}^{2}, \ldots, \sigma_{k}^{2}\right]\right)$ is the signal covariance matrix, where $(\bullet)^{H}$ denotes Hermitian transposition operator, $\operatorname{diag}(\bullet)$ denotes a diagonal matrix that uses the elements of a vector as its diagonal elements, $\sigma_{k}^{2}$ denotes the input signal power of the $k$ th signal, and $\sigma_{n}^{2}$ denotes the noise variance.

By vectorizing $\mathbf{R}_{\mathbf{x}}$, a new vector is obtained as

$$
\mathbf{z}=\operatorname{vec}\left(\mathbf{R}_{\mathbf{x}}\right)=\overline{\mathbf{A}} \mathbf{b}+\sigma_{n}^{2} \overline{\mathbf{I}},
$$

where $\operatorname{vec}(\bullet)$ denotes the vectorization operator that turns a matrix into a vector by stacking all columns on top of the other, and $\overline{\mathbf{A}}=\left[\overline{\mathbf{a}}\left(\theta_{1}\right), \ldots, \overline{\mathbf{a}}\left(\theta_{K}\right)\right], \mathbf{b}=\left[\sigma_{1}^{2}, \ldots, \sigma_{K}^{2}\right]^{T}$ and $\overline{\mathbf{I}}=$ $\operatorname{vec}\left(\mathbf{I}_{M+N-1}\right)$, where $\overline{\mathbf{a}}\left(\theta_{1}\right)=\mathbf{a}^{*}\left(\theta_{k}\right) \otimes \mathbf{a}\left(\theta_{k}\right),(\bullet)^{*}$ is the complex conjugation operator and $\otimes$ denotes the Kronecker product. Vector $\mathbf{z}$ amounts to the received data from a coherent source vector $\mathbf{b}$. The matrix $\overline{\mathbf{A}}$ behaves as the array manifold matrix of a virtual array with an extended aperture whose sensors are located at $p_{i}-p_{j}$ with $1 \leq i, j \leq M+N-1$. It has been shown in [5] that the choice of $L=\widetilde{M}+N$ yields the largest number of continuous lags for CADiS, and there are $M_{1}=2 M N+2 \widetilde{M}-1$ unique lags in the virtual array, among which the range $[(\widetilde{M}-1)(N-1), M N+\widetilde{M}-1]$ and its corresponding negative range are, respectively, continuous. In this paper, $\widetilde{M}>1$ is considered, and the virtual arrays of both coprime configurations are VSNLAs.

After removing repeated rows of (4) and sorting the remaining rows according to the unique lags set, the received source of the VSNLA is expressed as

$$
\widetilde{\mathbf{z}}=\widetilde{\mathbf{A}} \mathbf{b}+\sigma_{n}^{2} \mathbf{I}_{M_{1}},
$$

where $\widetilde{\mathbf{A}}=\left[\widetilde{\mathbf{a}}\left(\theta_{1}\right), \ldots, \widetilde{\mathbf{a}}\left(\theta_{K}\right)\right]$, and $\widetilde{\mathbf{a}}\left(\theta_{k}\right)=\left[e^{j 2 \pi \widetilde{p}_{1} \sin \left(\theta_{k}\right) / \lambda}, \ldots\right.$, $\left.e^{j 2 \pi \widetilde{p}_{M_{1}} \sin \left(\theta_{k}\right) / \lambda}\right]^{T}$ is $M_{1} \times 1$ steering vector, and $\widetilde{\mathbf{p}}=\left[\tilde{p}_{1}, \ldots\right.$, $\left.\tilde{p}_{M_{1}}\right]^{T}$ is the positions of the array sensors in the VSNLA. It is easy to verify that $\mathbf{I}_{M_{1}}$ is a vector whose elements are obtained by removing repeated elements of $\overline{\mathbf{I}}$ and sorting the remaining elements according to the unique lags set. Since the virtual signal in (5) becomes a single snapshot of $\mathbf{b}$, MUSIC algorithm fails to yield reliable DOA estimation when multiple sources imping to the array because that the rank of the noise-free covariance matrix $\mathbf{R}_{\widetilde{\mathbf{z}}}=\widetilde{\mathbf{z}} \widetilde{\mathbf{z}}^{H}$ of the VSNLA is one. The problem is similar to handling fully coherent sources. To solve this problem, spatial smoothing technique is applied to restore the rank of the covariance matrix [2]. Since spatial smoothing technique requires a filled ULA, data of continuous lag in the VSNLA is extracted [5], which means that only part of unique lags in the VSNLA can be used to implement the SS-MUSIC. Therefore, the maximum number of the sources that can be detected with this method is $\left\lfloor M_{c} / 2\right\rfloor$, where $M_{c}$ is the number of continuous lags and $\lfloor\bullet$ denotes the largest integer not exceeding the argument. The CADiS has $M_{c}=M N+\widetilde{M}-(\widetilde{M}-1)(N-1)$ continuous lags. Obviously, the virtual aperture offered by coprime array is not fully explored and the maximum number of detectable sources is lower than half that of the unique lags $\left\lfloor M_{1} / 2\right\rfloor$. It is noted that other kinds of coprime arrays with holes in their virtual array have the same problem.

\section{DOA Estimation with Array Interpolation Based on Compressing Sensing}

The usage of SS-MUSIC for the coprime array only exploits the continuous lag set in the VSNLA of a coprime array, resulting in relatively poor DOA estimation precision and low number of resolvable sources. To explore the whole aperture of the VSNLA for DOA estimation, CS-based methods such as OMP and LASSO can be used, while such methods suffer from basis mismatch effects. The sparsity-based extrapolation technique [6] uses sparse reconstruction to extrapolate observations at the holes in the VSNLA and then utilizes SSMUSIC to obtain the final DOA estimation. However, some of the measurements may not be accurately reconstructed due to basis mismatch effects. The coarray interpolation using nuclear norm minimization in [17] has no basis mismatch problem, while it requires a complex semidefinite programming solver. To overcome basis mismatch problem and obtain a relatively low computational complexity, a compressing sensing based array interpolation approach is proposed in this section, like the sparsity-based extrapolation technique, CS reconstruction is also used in our proposed method, while it is used to obtain the initial DOA estimation. We first review the conventional iterative initial DOA estimation based array interpolation and the LASSO compressing sensing technique. Then, in Section 3.2, we introduce the compressing sensing based array interpolation approach, which uses the LASSO compressing sensing technique to obtain the coarse initial DOA estimation. Finally, we outline the proposed method by joining the main procedures together. 
3.1. Conventional Iterative Initial DOA Estimation Based Array Interpolation for Coprime Array. The key idea of the initial DOA estimation based array interpolation approach in [22] is to design an appropriate mapping matrix $\mathbf{B}$. The mapping matrix is then used to map the data of the VSNLA of the coprime to that of a virtual filled ULA with the same aperture over a union of particular interpolation sectors $\left\{\boldsymbol{\theta}_{k}\right\}$ that covers all the sources inside. Suppose that $\widetilde{\mathbf{a}}(\theta)$ and $\widetilde{\mathbf{a}}^{\prime}(\theta)$ are array steering vectors for the VSNLA of coprime array and the virtual filled ULA (i.e., a filled VSNLA with the same aperture size as the VSNLA), respectively. The interpolation matrix $\mathbf{B}$ should obey

$$
\widetilde{\mathbf{a}}^{\prime}(\theta)=\mathbf{B} \widetilde{\mathbf{a}}(\theta), \quad \theta \in \boldsymbol{\theta}_{k} .
$$

It is noted that there is no guarantee that a signal outside interpolation sectors will be identified correctly, because signal outside of the interpolation sector will cause large interpolation errors. Therefore, initial DOA estimation $\widehat{\boldsymbol{\theta}}=\left[\widehat{\theta}_{1}, \ldots, \widehat{\theta}_{K}\right]^{T}$ is required to locate these particular interpolation sectors. After that, the initial DOA estimation is obtained, each small interpolation sector $\boldsymbol{\theta}_{k}=\left[\widehat{\theta}_{k}-\right.$ $\left.\theta_{\varepsilon}, \widehat{\theta}_{k}+\theta_{\varepsilon}\right]$ that contains the $k$ th signal inside is uniformly divided with $\delta \theta$ intervals, and array manifolds $\widetilde{\mathbf{A}}(\bar{\theta})$ and $\widetilde{\mathbf{A}}^{\prime}(\bar{\theta})$ for the VSNLA and the virtual filled ULA are generated by considering $\bar{\theta}_{i}=i \delta \theta\left(i=1, \ldots, 2 \theta_{\varepsilon} / \delta \theta+1\right)$, respectively. To improve the DOA performance for noisy observations, the mapping matrix of the Wiener solution is then given as [22]

$$
\mathbf{B}=\sigma_{s}^{2} \widetilde{\mathbf{A}}^{\prime}(\bar{\theta}) \widetilde{\mathbf{A}}(\bar{\theta})^{H}\left(\widetilde{\mathbf{A}}(\bar{\theta}) \widetilde{\mathbf{A}}(\bar{\theta})+\sigma_{n}^{2} \mathbf{I}\right)^{-1},
$$

where $\sigma_{s}^{2}$ is the average power of sources.

As discussed in [23], if the width of the interpolation sector, $2 \theta_{\varepsilon}$ is too wide, the interpolation error may be unacceptable. To overcome this problem, we set the width of the interpolation sector to be equal to half of the main beam width of the VSNLA. The interval size $\delta \theta$ is chosen experimentally as described in [21].

The conventional iterative initial DOA estimation based array interpolation method, IMCA-AI [22], is applied to the VSNLA of coprime array for DOA estimation of sources as follows.

Step 1. Obtain the estimation of the covariance matrix of the real array $\mathbf{R}_{\mathbf{x}}$ :

$$
\widehat{\mathbf{R}}_{\mathbf{x}}=\frac{1}{T} \sum_{t=1}^{T} \mathbf{x}(t) \mathbf{x}^{H}(t) .
$$

Step 2. Get vector $\widetilde{\mathbf{z}}$ of the VSNLA from (4) and (5), and find the estimation of the covariance matrix of the VSNLA $\widehat{\mathbf{R}}_{\widetilde{\mathbf{z}}}$ :

$$
\widehat{\mathbf{R}}_{\widetilde{\mathbf{z}}}=\widetilde{\mathbf{z}} \widetilde{\mathbf{z}}^{H}
$$

Step 3. Extract the output samples of one continuous lags set of the VSNLA $\widetilde{\mathbf{z}}_{1}$ from $\widetilde{\mathbf{z}}$ to find the covariance matrix estimation $\widehat{\mathbf{R}}_{\widetilde{\mathbf{z}}_{1}}$ for spatial smoothing:

$$
\widehat{\mathbf{R}}_{\widetilde{\mathbf{z}}_{1}}=\widetilde{\mathbf{z}}_{1} \widetilde{\mathbf{z}}_{1}^{H}
$$

Step 4. Then apply the SS-MUSIC algorithm to $\widehat{\mathbf{R}}_{\widetilde{\mathbf{z}}_{1}}$ for the coarse initial DOA estimate $\widehat{\boldsymbol{\theta}}$.

Step 5. Given $\widehat{\boldsymbol{\theta}}$, construct B from (7).

Step 6. Find the covariance matrix estimate of the virtual filled VSULA $\widetilde{\mathbf{R}}_{\widetilde{\mathbf{z}}}^{\prime}=\mathbf{B} \widehat{\mathbf{R}}_{\widetilde{\mathbf{z}}} \mathbf{B}^{H}$, and use the forward-backward spatial smoothing to find the DOA by the MUSIC algorithm.

Step 7. Use the estimated DOA in Step 4 and repeat Steps from 3 to 6 until the preset maximal number of iterations $K_{\max }\left(K_{\max } \geq 3\right)$ is reached.

According to the above steps, the whole aperture of the VSNLA of the coprime array is used to get the DOA estimates after using array interpolation, which will significantly improve the DOA estimation precision. However, the key part of the IMCA-AI lies on the assumption that the reliable initial DOA estimates $\widehat{\boldsymbol{\theta}}$ will be obtained in Step 4 with continuous lags in the VSNLA. When the number of sources is higher than $\left\lfloor M_{c} / 2\right\rfloor$, the SS-MUSIC algorithm fails to get the roughly initial DOA estimates of sources, thus causing unacceptable interpolation error and big DOA estimation bias. Therefore, the maximum number of resolvable sources using IMCA-AI is equal to half the number of continuous lags in the VSNLA.

3.2. Compressing Sensing for Coprime Array. Alternatively, (5) can be solved using the CS algorithm [5, 7, 9]. By discretizing the entire angular space from $-90^{\circ}$ to $90^{\circ}$ as a grid $\boldsymbol{\Theta}=\left[\theta_{1}, \ldots \theta_{D}\right]^{T}$ with $\theta_{l+1}-\theta_{l}=180^{\circ} /(D-1)$, for $l=1, \ldots, D$, where $D \gg K$, the model in (5) can be transformed into

$$
\widetilde{\mathbf{z}}=\widetilde{\mathbf{A}}^{\circ} \mathbf{b}^{\circ}+\sigma_{n}^{2} \mathbf{I}_{M_{1}}=\mathbf{B}^{\circ} \widetilde{\mathbf{b}}^{\circ}
$$

where $\widetilde{\mathbf{A}}^{\circ}=\left[\widetilde{\mathbf{a}}\left(\theta_{1}\right), \ldots, \widetilde{\mathbf{a}}\left(\theta_{D}\right)\right]$ is the overcomplete basis matrix for $\widetilde{\mathbf{A}}$ parameterized by all the directions in set $\Theta$ and $\mathbf{b}^{\circ}$ is the sparse representation of the source power vector in which only the positions of entries corresponding to the actual DOAs are occupied by nonzero entries. In addition, $\mathbf{B}^{\circ}=\left[\widetilde{\mathbf{A}}^{\circ}, \mathbf{I}_{M_{1}}\right]$, and $\widetilde{\mathbf{b}}^{\circ}=\left[\mathbf{b}^{\circ}, \sigma_{n}^{2}\right]^{T}$. Therefore, signal $\widetilde{\mathbf{b}}^{\circ}$ is $K+1$ sparse in this setting. If $\widetilde{\mathbf{b}}^{\circ}$ can be recovered from (11), the DOAs and $\sigma_{n}^{2}$ can be identified from the nonzero entries of $\widetilde{\mathbf{b}}^{\circ}$.

Given the model in (11), DOA estimation proceeds in terms of sparse signal reconstruction by solving the following constrained minimization problem:

$$
\begin{aligned}
\widehat{\mathbf{b}}^{\circ}=\arg \underset{\widetilde{\mathbf{b}}^{\circ}}{\operatorname{minimize}} & \left\|\mathbf{b}^{\circ}\right\|_{1} \\
\text { subject to } & \left\|\widetilde{\mathbf{z}}-\mathbf{B}^{\circ} \widetilde{\mathbf{b}}^{\circ}\right\|_{2}<\varepsilon,
\end{aligned}
$$

where $\varepsilon$ is a user-specified bound which depends on the noise variance. This type of problem has been the objective of intensive studies in the area of CS, and various techniques can be used to solve the constrained minimization problem in (12), such as LASSO, BP, and OMP [7, 8, 26, 27]. In 
$[9,28,29]$, the LASSO algorithm was used to solve an equivalent problem to (12):

$$
\widehat{\mathbf{b}}^{\circ}=\arg \underset{\widetilde{\mathbf{b}}^{\circ}}{\operatorname{minimize}}\left[\frac{1}{2}\left\|\widetilde{\mathbf{z}}-\mathbf{B}^{\circ} \widetilde{\mathbf{b}}^{\circ}\right\|_{2}+\lambda_{t}\left\|\widetilde{\mathbf{b}}^{\circ}\right\|_{1}\right],
$$

where $\lambda_{t}$ is a penalty parameter used to control the weight of the sparsity constraint in the overall cost function. According to [28], the maximum number of resolvable sources using LASSO equals the number of unique positive lags in the VSNLA of coprime array, that is, $\left\lfloor M_{1} / 2\right\rfloor$. However, it should be noted that the sparse model (11) is exact only when all the sources are located exactly on points in the grid set $\Theta$; otherwise, the compressed sensing suffers from dictionary mismatches and the performance is deteriorated [30].

\subsection{Proposed Array Interpolation Based on Compressed Sens-} ing for Coprime. From above analysis, we see that IMCIAI has better DOA estimation accuracy than the SS-MUSIC algorithm. However, the maximum number of resolvable sources of these two methods is relatively smaller when compared with compressed sensing algorithm like LASSO. Since the true DOAs are unlikely to lie on the prespecified grid, no matter how finely it is chosen, the LASSO algorithm suffers from basis mismatch effects. A simple but effective way to improve the estimation accuracy and increase the maximum number of resolvable sources is to combine both advantages of IMCI-AI and the LASSO algorithm. Here, we propose a modified array interpolation based on compressed sensing for coprime array with holes in its virtual array. The proposed method uses LASSO to obtain the initial DOA estimate for iterative initial DOA estimation based array interpolation. Though basis mismatch may deteriorate the reconstruction performance of LASSO, the result of LASSO with bias can still be used as initial DOA estimate for the proposed method. The approach proposed for the VSNLA of coprime array is given as follows.

Step 1. Obtain the estimation of the covariance matrix of the real array $\mathbf{R}_{\mathbf{x}}$.

Step 2. Use vector $\widetilde{\mathbf{z}}$ of the VSNLA from (4) and (5), and find the estimation of the covariance matrix of the VSNLA $\widehat{\mathbf{R}}_{\tilde{\mathbf{z}}}$.

Step 3. Apply the LASSO algorithm to $\widetilde{\mathbf{z}}$ for the coarse initial DOA estimate $\widehat{\boldsymbol{\theta}}$.

Step 4. Given $\widehat{\boldsymbol{\theta}}$, construct B from (7).

Step 5. Find the covariance matrix estimate of the virtual filled ULA $\widehat{\mathbf{R}}_{\tilde{\mathbf{z}}}^{\prime}=\mathbf{B} \widehat{\mathbf{R}}_{\tilde{\mathbf{z}}} \mathbf{B}^{H}$, and use the ESPRIT-Like algorithm proposed in [25] for coherent DOA estimation.

Step 6. Use estimated DOA in Step 5 and repeat Steps 3 and 4 until the preset maximal number of iterations $K_{\max }\left(K_{\max } \geq\right.$ 3 ) is reached.

Remark 1. Since the estimation of $\sigma_{n}^{2}$ for the VSNLA and the estimation of the average power of sources $\sigma_{s}^{2}$ in $\widetilde{\mathbf{z}}$ are required in (7) for the construction of the map matrix $\mathbf{B}$.
TABLE 1: Comparison of the four methods for coprime array.

\begin{tabular}{lcccc}
\hline \multicolumn{2}{c}{ SS-MUSIC IMCA-AI } & LASSO & $\begin{array}{c}\text { The } \\
\text { proposed } \\
\text { method }\end{array}$ \\
\hline $\begin{array}{l}\text { Maximum number } \\
\text { of resolvable sources }\end{array}$ & $\left\lfloor M_{c} / 2\right\rfloor$ & $\left\lfloor M_{c} / 2\right\rfloor$ & $\left\lfloor M_{1} / 2\right\rfloor$ & $\left\lfloor M_{1} / 2\right\rfloor$ \\
Accuracy & Low & High & $\begin{array}{c}\text { Low } \\
\text { (off-grid } \\
\text { source) }\end{array}$ & High \\
\hline
\end{tabular}

The eigenvalue decomposition of the covariance matrix of the VSNLA $\widehat{\mathbf{R}}_{\tilde{\mathbf{z}}}$ is used to yield

$$
\widehat{\mathbf{R}}_{\widetilde{\mathbf{z}}}=\sum_{m}^{M_{1}} \lambda_{m} \mathbf{e}_{m} \mathbf{e}_{m}^{H},
$$

where $\lambda_{1} \geq \lambda_{2} \geq \cdots \geq \lambda_{K} \geq \cdots \geq \lambda_{M_{1}}$ are the eigenvalues of $\widehat{\mathbf{R}}_{\widetilde{\mathbf{z}}}$ and $\mathbf{e}_{m}\left(m=1, \ldots, M_{1}\right)$ are corresponding eigenvectors. According to [31], the estimate of noise variance $\sigma_{n}^{2}$ can be obtained by averaging the $M_{1}-K$ smallest eigenvalues. Similarly, the estimation of the average power of sources $\sigma_{s}^{2}$ can also be obtained by averaging the $K$ largest eigenvalues.

Remark 2. The filled virtual ULA after interpolation using data of VSNLA is actually a virtual filled VSULA. The VSULA of CADiS has a total number of $M_{\alpha}=2(M N+\widetilde{M} N-\widetilde{M}-$ $2 N+L)+1$ lags. The ESPRIT-Like algorithm [25] is used instead of MUSIC with forward-backward spatial smoothing for its simple computation and robust DOA estimation performance. Compared with LASSO, the proposed method can detect up to $\left\lfloor M_{1} / 2\right\rfloor$ sources with the filled VSULA, while it has no problem of basis mismatch problem. Table 1 summarizes the number of resolvable sources and angle accuracy using four methods.

Remark 3. The reliable coarse initial DOA estimate obtained using Step 3 is essential for the success of the subsequent DOA estimation. And it is noted that the source number $K$ is assumed to be known as a priori in this paper. The $K$ largest peaks in the spectrum of LASSO are taken as detected sources. If the initial estimate has missed detections, the obtained small interpolation sectors fail to cover the missed sources inside, which leads to big interpolation errors in the directions of the missed sources, thus causing big DOA estimate bias for the missed sources, while these detected sources that are covered in the obtained small interpolation sectors can still be detected with increased DOA estimation bias. If the initial estimate using LASSO has spurious peaks with larger amplitude than that of true sources, the spurious peaks may be wrongly taken as sources while true sources with smaller amplitude will be lost. As a result, redundant interpolation sectors that cover the spurious peaks inside are used in the designed interpolation sectors, and the missed sources are not covered, which also leads to big interpolation errors. 


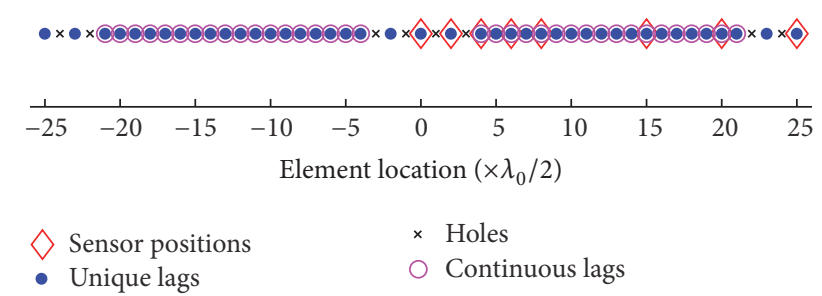

Figure 2: The VSNLA of a CADiS coarray with $M=4, N=5$, $p=2, \widetilde{M}=2$, and $L=7$.

\section{Simulation Results}

In this section, computer simulations are performed to evaluate the proposed approach. Since the CADiS and other coprime array with holes in their SVNLA structures have the same problem. For simplicity, we only use the CADiS coprime array in the following simulations to demonstrate the advantages of the proposed method. A CADiS configuration with $M=4, N=5$ and $p=2$ is considered and the displacement between the two subarrays is set as $L=\widetilde{M}+N=7$. This configuration has $M+N-1=8$ physical antenna sensors and the VSNLA corresponding to the CADiS is shown in Figure 2. The VSNLA has $M_{1}=43$ unique lags, among which, lags within $[-21,-4]$ and $[4,21]$ are, respectively, continuous. After interpolation, the filled VSULA has $M_{\alpha}=51$ lags. Other existing methods are also listed for comparison, including SS-MUSIC [2], LASSO [5], IMCA-AI [22], sparsity-based extrapolation method (Sparse MUSIC) [6], coprime coarray interpolation technique using nuclear norm minimization (nuclear norm) [17], the offgrid DOA estimation method using sparse Bayesian inference (OGSBI) [13], and joint LASSO (JLASSO) [12]. The number of achievable DoFs is 9 for SS-MUSIC and IMCA-AI, while the number of achievable DoFs is 21 for LASSO, Sparse MUSIC, JLASSO, OGSBI, and the proposed approach. We take the grid from $-90^{\circ}$ to $90^{\circ}$ with step size $1^{\circ}$ to perform the proposed array interpolation method with off-grid sources. We perform both IMCA-AI and the proposed method with the maximum number of iterations $K_{\max }=4$, and $\theta_{\varepsilon}=$ $\left[1^{\circ}, 0.5^{\circ}, 0.25^{\circ}, 0.125^{\circ}\right]$ is used at each iteration. The parameter $\lambda_{t}$ used in LASSO is normally chosen by cross-validation [32]. These simulations are performed on a Windows 10 workstation with Intel Core i7-2600 3.4 GHz processor and 14 GB RAM.

4.1. Resolution Tests. In the first simulation, we test the resolution ability by detecting two closely located sources. The two off-grid uncorrelated narrowband sources are located at $-31.45^{\circ}$ and $-28.65^{\circ}$, respectively. To compare the performance among the aforementioned eight methods, $T=500$ snapshots are used. We perform the experiment with signalto-noise ratio (SNR) that is equal to $0 \mathrm{~dB}$ and $-10 \mathrm{~dB}$ for both sources with $\lambda_{t}=0.25$ and $\lambda_{t}=0.035$ for LASSO, respectively. The underlying DOAs are denoted in the figures by red dashed lines. We use black circle without amplitude to denote the DOA estimations of the proposed method for convenience in the following figures including Figures 3, 4, 6, and 8.
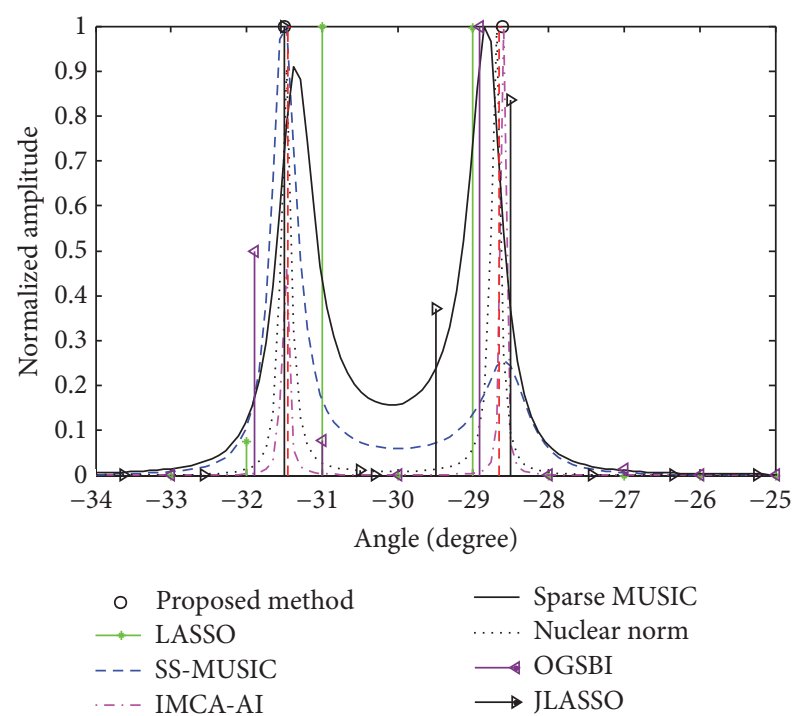

FIgURE 3: Resolution performance (SNR $\left.=0 \mathrm{~dB}, \lambda_{t}=0.25\right)$.

We can see from Figure 3 that both sources can be separated using all eight methods when $\mathrm{SNR}=0 \mathrm{~dB}$, while the proposed method, IMCA-AI, Sparse MUSIC, and nuclear norm have better resolution performance than SS-MUSIC because the whole aperture of VSNLA is used after using array interpolation. For the LASSO algorithm, relatively big DOA bias is caused by the dictionary mismatch effects; however, it can be used for the coarse initial DOA for the proposed method. Though the DOA estimations of OGSBI and JLASSO are closer to the true source DOA than that of LASSO, the proposed method has better estimation than OGSBI and JLASSO. Figure 4 shows that SS-MUSIC fails to separate two sources when SNR equals $-10 \mathrm{~dB}$, which also leads to the failure of IMCA-AI because the coarse initial DOAs of two sources are not obtained, only one source is detected with big bias, the obtained interpolation sector fails to cover both sources inside. However, other six algorithms succeed in separating both sources.

We compute the CPU time to run the eight algorithms on the same data for a quantitative comparison. The results are tabulated in Table 2. LASSO takes the shortest time while JLASSO takes the longest time because the time-consuming convex optimization is required. Nuclear norm also has the same problem with JLASSO in solving the reconstruction problem. The proposed method takes the second shortest time because ESPRIT-Like algorithm that avoids the spatial search is used in the last stage for the final DOA estimation. SS-MUSIC takes longer time than the proposed method because it uses spatial search. Sparse MUSIC takes longer time than LASSO and SS-MUSIC because it uses both LASSO and SS-MUSIC in the first and second stage, respectively. IMCA-AI and OGSBI take comparable time.

4.2. Detect Performance. In the second numerical experiment, we demonstrate that the proposed array interpolation method can detect more targets than SS-MUSIC and IMCAAI. We consider $K=9$ off-grid narrowband sources, which 
TABLE 2: CPU times of the eight methods.

\begin{tabular}{lcccccccc}
\hline Algorithm & LASSO & Proposed method & SS-MUSIC & Sparse MUSIC & IMCA-AI & OGSBI & Nuclear norm & JLASSO \\
\hline CPU time $(\mathrm{s})$ & 0.0118 & 0.0724 & 0.0940 & 0.0971 & 0.5049 & 0.5386 & 2.2018 & 3.6824 \\
\hline
\end{tabular}
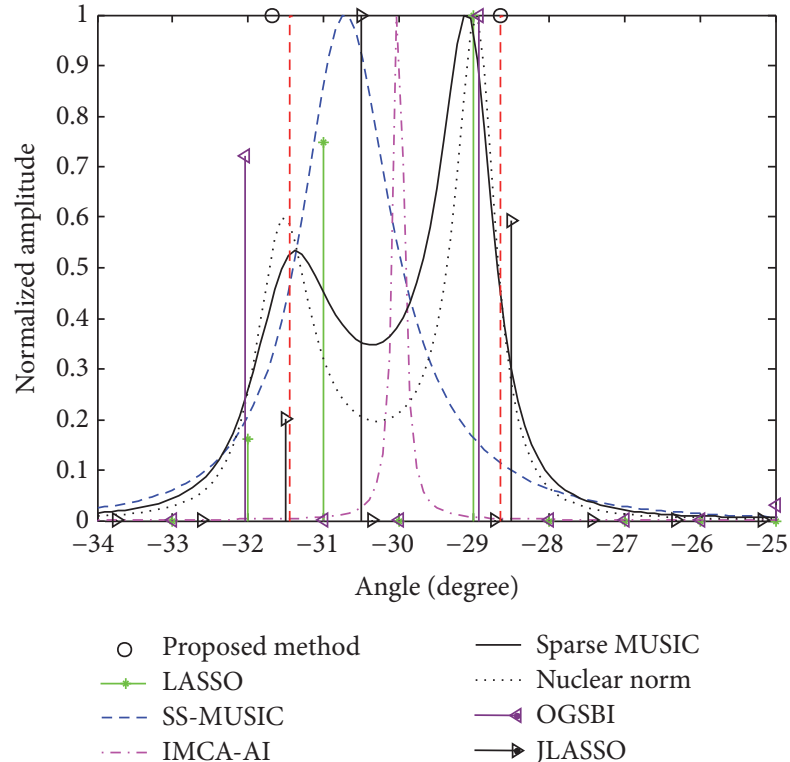

FIGURE 4: Resolution performance $\left(\mathrm{SNR}=-10 \mathrm{~dB}, \lambda_{t}=0.035\right)$.

is the maximum number that SS-MUSIC and IMCA-AI can detect theoretically. The 9 sources are uniformly distributed between $-73.2^{\circ}$ and $70.3^{\circ}$. The number of snapshots is $T=$ 500 , and the SNR is set to be $0 \mathrm{~dB}$.

We can see from Figure 5 that one source is lost using both SS-MUSIC and IMCA-AI, while Sparse MUSIC and nuclear norm succeed in detecting all 9 sources. The proposed method, LASSO, OGSBI, and JLASSO can detect all 9 sources as shown in Figure 6, which is more than the number of physical sensors.

To further demonstrate that the proposed method can detect more targets, we consider $K=14$ off-grid uncorrelated narrowband sources, which are uniformly distributed between $-73.2^{\circ}$ and $70.3^{\circ}$, and other simulation parameters are set as above. In this setting, SS-MUIC is unable to produce a spectrum because the number of sources exceeds its limit. Therefore, IMCA-AI also fails because the initial DOA estimations are not obtained using SS-MUSIC. Figure 7 only shows the spatial spectrums of Sparse MUSIC and nuclear norm, which shows that both methods succeed in detecting all sources. Figure 8 shows that JLASSO misses several sources, while the proposed method, LASSO, and OGSBI can also detect all 14 sources.

4.3. Estimation Accuracy. In the next experiment, we further compare the DOA estimation accuracy of all eight methods through 500 Mont Carlo trials by estimation $K=2$ uncorrelated narrowband sources with an angle separation of $10^{\circ}$, and their directions are $-5^{\circ}+\varphi$ and $5^{\circ}+\varphi$, respectively,

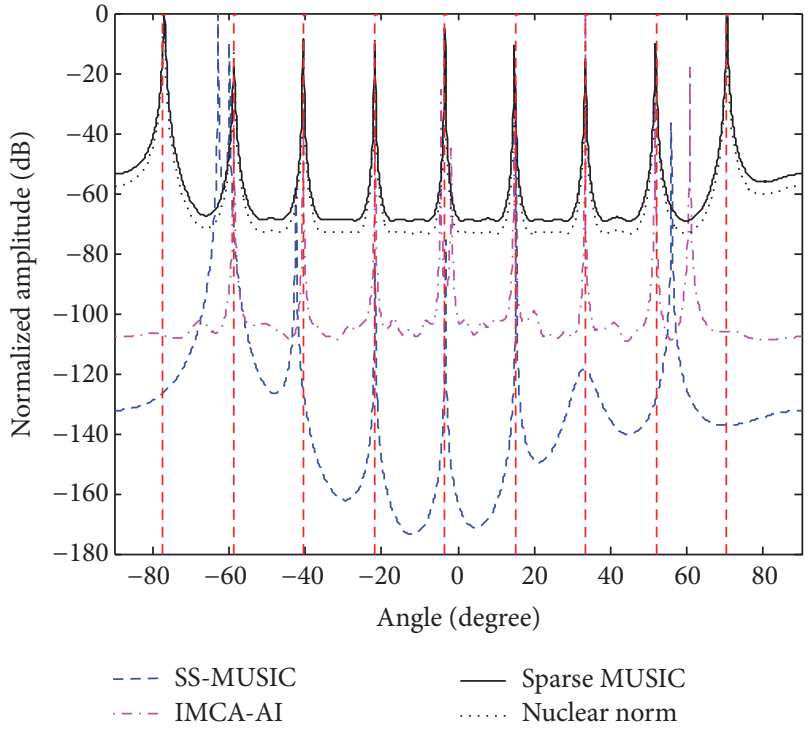

FIGURE 5: Spatial spectrums of MUSIC, IMCA-AI, Sparse MUSIC, and nuclear norm $(K=9)$.

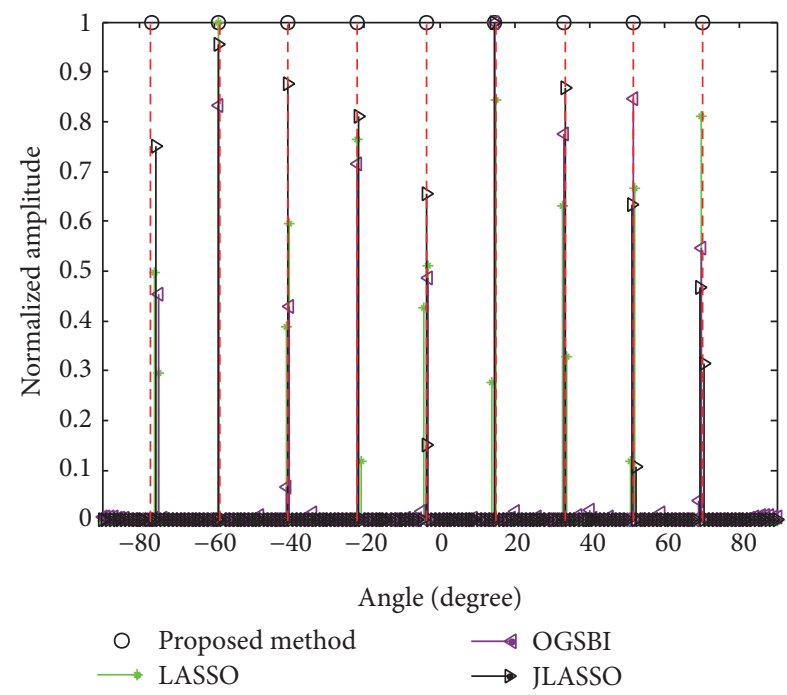

FIGURE 6: Spatial spectrums of LASSO, OGSBI, and the DOA results of the proposed method $\left(K=9, \lambda_{t}=0.25\right)$.

with $\varphi$ chosen uniformly and randomly in the range from $-0.5^{\circ}$ to $0.5^{\circ}$ in each trial. The average root mean square error (RMSE) of the DOA estimate is used as the performance metric, which is index written by

$$
\operatorname{RMSE}=\sqrt{\frac{1}{500 K} \sum_{m=1}^{500} \sum_{i=1}^{K}\left[\vartheta_{i}(m)-\theta_{i}\right]^{2}}
$$




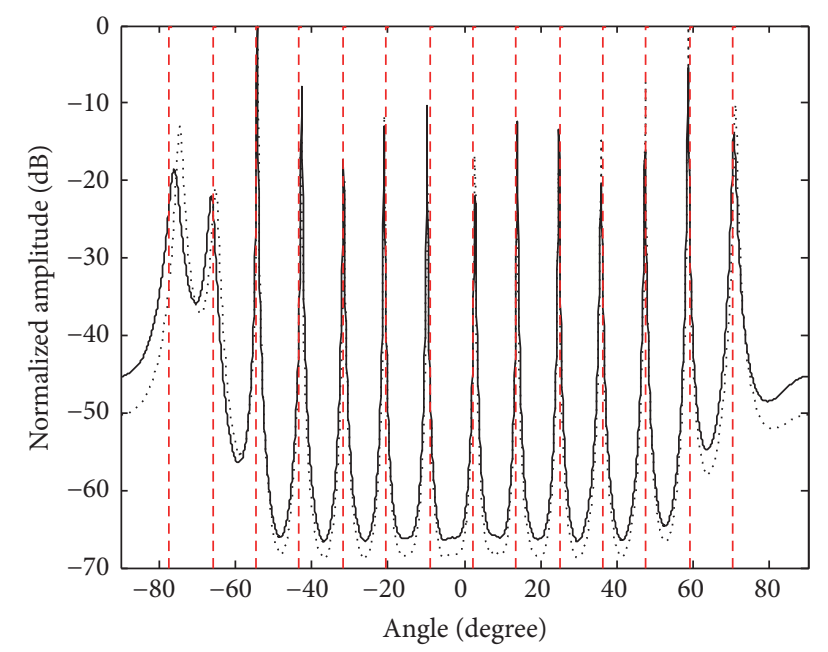

- Sparse MUSIC

FIgURE 7: Spatial spectrums of Sparse MUSIC and nuclear norm $(K=14)$.

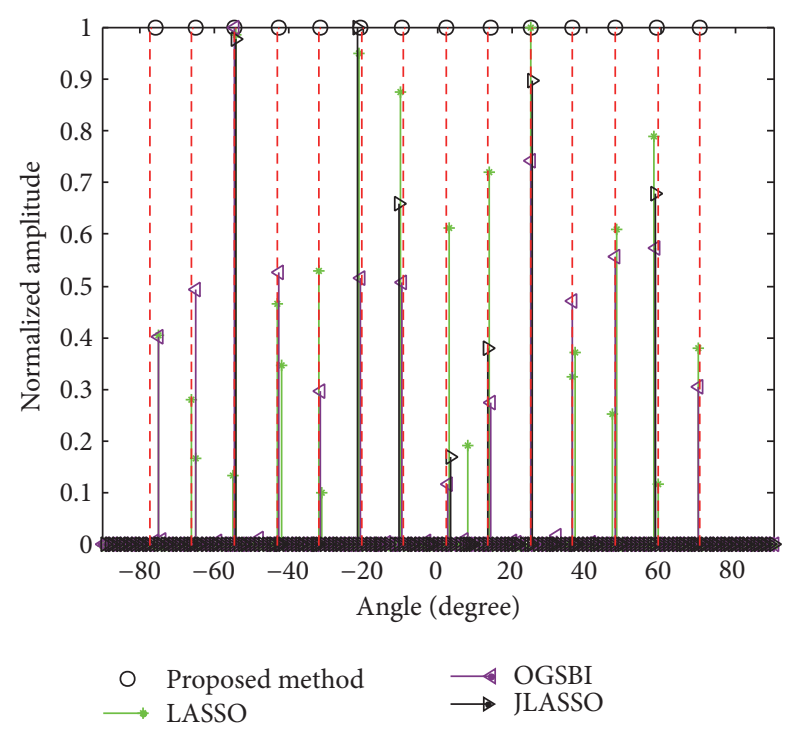

FIGURE 8: Spatial spectrums of LASSO, OGSBI, and the DOA results of the proposed method $\left(K=14, \lambda_{t}=0.25\right)$.

where $\vartheta_{i}(m)$ denotes the estimate of $\theta_{i}$ for the $m$ th Monte Carlo trial. The number of snapshots is $T=300$. Figure 9 compares the RMSE performance as a function of the input SNR. We can see that the performance of LASSO does not improve with the increase of SNR due to basis mismatch effects. OGSBI has better performance than LASSO when SNR is above $1 \mathrm{~dB}$, but it has bigger RMSE than other six methods when SNR is below $1 \mathrm{~dB}$. Because IMCA-AI, Sparse MUSIC, nuclear norm, and the proposed method have made the most of the array aperture of the VSNLA after interpolation, they all have better performance than SS-MUSIC. The proposed method and IMICA-AI have better performance than two interpolation methods, while the proposed method

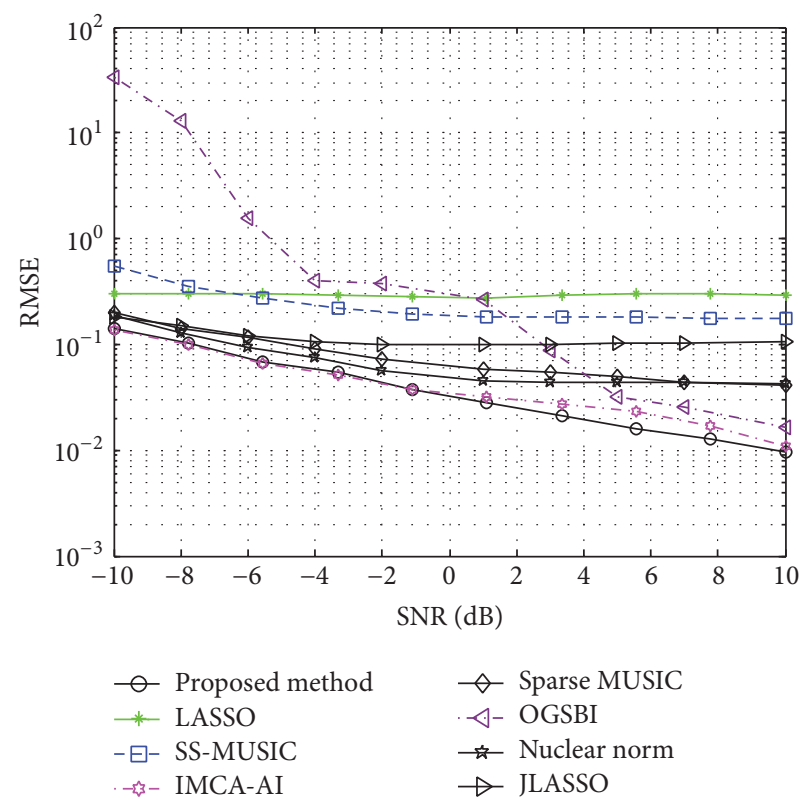

Figure 9: RMSE versus SNR $(T=300, K=2)$.

achieves higher precision than IMCA-AI when SNR is above $0 \mathrm{~dB}$.

In the last experiment, we fix the angle separation of the two sources at $10^{\circ}$ and their SNR at $0 \mathrm{~dB}$. When the snapshot number increases from 50 to 5000 , the averaged DOA estimation RMSE curves of the all eight methods are shown in Figure 10. The proposed method and IMCA-AI have almost the same better performance than other six methods when the snapshots are more than about 550, while the proposed method has the lowest RMSE when the snapshot is low. LASSO has better performance than IMCA-AI and SS-MUSIC when the number of snapshot is low, while the performance of LASSO method does not improve with the increase of snapshots due to the basis mismatch effects. The OGBSI has slightly better performance than LASSO, while JLASSO has better performance than OGBSI. As the snapshot number increases, the DOA estimation precision of SSMUSIC, Sparse MUSIC, and nuclear norm improves slowly, and these three methods have almost the same precision. However, both IMCA-AI and the proposed method surpass the other six methods.

\section{Conclusion}

A compressed sensing based array interpolation approach that uses the DOA estimation from compressing sensing method as initial DOA estimation is proposed to fill the holes in the VSNLA of coprime array, which can obtain a filled VSULA with more DoFs when the ESPRIT-Like algorithm is used. The proposed method can also be used to overcome the off-grid problem of CS. In addition, the proposed method can solve more sources compared with conventional initial DOA estimation based array interpolation method. Simulation results show the validity and efficiency of our proposed method. 


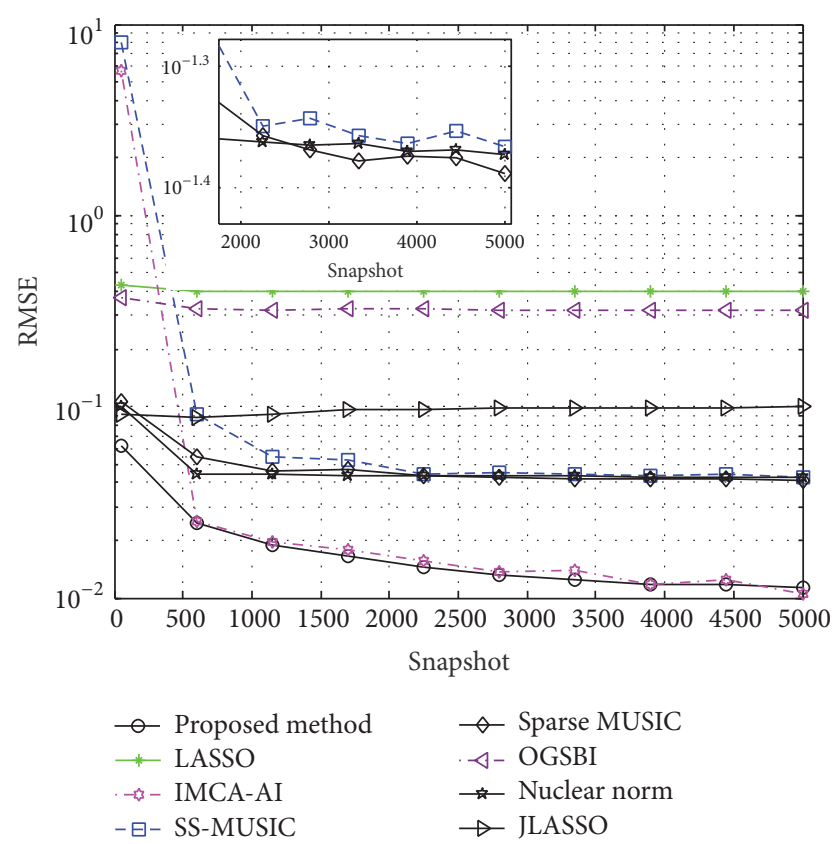

FIgURE 10: RMSE versus snapshot $(\mathrm{SNR}=0, K=2)$.

\section{Competing Interests}

The authors declare that there is no conflict of interests regarding the publication of this paper.

\section{Acknowledgments}

This work was supported by the National Natural Science Foundation of China under Grant no. 611711802 and no. 61032011.

\section{References}

[1] R. O. Schmidt, "Multiple emitter location and signal parameter estimation," IEEE Transactions on Antennas and Propagation, vol. 34, no. 3, pp. 276-280, 1986.

[2] P. Pal and P. P. Vaidyanathan, "Nested arrays: a novel approach to array processing with enhanced degrees of freedom," IEEE Transactions on Signal Processing, vol. 58, no. 8, pp. 4167-4181, 2010.

[3] P. P. Vaidyanathan and P. Pal, "Sparse sensing with co-prime samplers and arrays," IEEE Transactions on Signal Processing, vol. 59, no. 2, pp. 573-586, 2011.

[4] P. Pal and P. P. Vaidyanathan, "Coprime sampling and the music algorithm," in Proceedings of the IEEE Digital Signal Processing Workshop and IEEE Signal Processing Education Workshop (DSP/SPE '11), pp. 289-294, Napa, Calif, USA, 2011.

[5] S. Qin, Y. D. Zhang, and M. G. Amin, "Generalized coprime array configurations for direction-of-arrival estimation," IEEE Transactions on Signal Processing, vol. 63, no. 6, pp. 1377-1390, 2015.

[6] E. BouDaher, F. Ahmad, and M. G. Amin, "Sparsity-based extrapolation for direction-of-arrival estimation using coprime arrays," in Compressive Sensing V: From Diverse Modalities to Big Data Analytics, F. Ahmad, Ed., vol. 9857 of Proceedings of SPIE, Baltimore, Md, USA, April 2016.
[7] J. A. Tropp and A. C. Gilbert, "Signal recovery from random measurements via orthogonal matching pursuit," Transactions on Information Theory, vol. 53, no. 12, pp. 4655-4666, 2007.

[8] R. Tibshirani, "Regression shrinkage and selection via the lasso," Journal of the Royal Statistical Society. Series B. Methodological, vol. 58, no. 1, pp. 267-288, 1996.

[9] Y. D. Zhang, M. G. Amin, and B. Himed, "Sparsity-based DOA estimation using co-prime arrays," in Proceedings of the IEEE International Conference on Acoustics, Speech and Signal Processing (ICASSP '13), pp. 3967-3971, IEEE, Vancouver, Canada, May 2013.

[10] P. Pal and P. P. Vaidyanathan, "On application of LASSO for sparse support recovery with imperfect correlation awareness," in Proceedings of the 46th Asilomar Conference on Signals, Systems and Computers (ASILOMAR '12), pp. 958-962, Pacific Grove, Calif, USA, November 2012.

[11] S. Qin, Y. D. Zhang, and M. G. Amin, "Generalized coprime array configurations," in Proceedings of the IEEE 8th Sensor Array and Multichannel Signal Processing Workshop (SAM '14), pp. 529-532, A Coruna, Spain, June 2014.

[12] Z. Tan and A. Nehorai, "Sparse direction of arrival estimation using co-prime arrays with off-grid targets," IEEE Signal Processing Letters, vol. 21, no. 1, pp. 26-29, 2014.

[13] Z. Yang, L. Xie, and C. Zhang, "Off-grid direction of arrival estimation using sparse Bayesian inference," IEEE Transactions on Signal Processing, vol. 61, no. 1, pp. 38-43, 2013.

[14] A. J. Weiss and B. Friedlander, "Performance analysis of spatial smoothing with interpolated arrays," in Proceedings of the International Conference on Acoustics, Speech, and Signal Processing (ICASSP '91), vol. 2, pp. 1377-1380, San Francisco, Calif, USA, May 1991.

[15] A. J. Weiss and B. Friedlander, "Performance analysis of spatial smoothing with interpolated arrays," IEEE Transactions on Signal Processing, vol. 41, no. 5, pp. 1881-1892, 1993.

[16] E. BouDaher, Y. Jia, F. Ahmad, and M. G. Amin, "Multifrequency co-prime arrays for high-resolution direction-ofarrival estimation," IEEE Transactions on Signal Processing, vol. 63, no. 14, pp. 3797-3808, 2015.

[17] C.-L. Liu, P. P. Vaidyanathan, and P. Pal, "Coprime coarray interpolation for DOA estimation via nuclear norm minimization," in Proceedings of the IEEE International Symposium on Circuits and Systems (ISCAS '16), pp. 2639-2642, May 2016.

[18] B. Friedlander and A. J. Weiss, "Direction finding using spatial smoothing with interpolated arrays," IEEE Transactions on Aerospace and Electronic Systems, vol. 28, no. 2, pp. 574-587, 1992.

[19] B. Friedlander and A. J. Weiss, "Direction finding for wideband signals using an interpolated array," in Proceedings of the Conference Record of the 25th Asilomar Conference on Signals, Systems and Computers, vol. 1, pp. 583-587, Pacific Grove, Calif, USA, November 1991.

[20] B. Friedlander and A. J. Weiss, "Performance analysis of wideband direction finding using interpolated arrays," in Proceedings of the IEEE International Conference on Acoustics, Speech, and Signal Processing (ICASSP '92), vol. 4, pp. 457-460, 1992.

[21] B. Friedlander, "The root-MUSIC algorithm for direction finding with interpolated arrays," Signal Processing, vol. 30, no. 1, pp. 15-29, 1993.

[22] T. E. Tuncer, T. K. Yasar, and B. Friedlander, "Direction of arrival estimation for nonuniform linear arrays by using array interpolation," Radio Science, vol. 42, no. 4, Article ID RS4002, 2007. 
[23] B. Li, B. Xu, and Y. Yuan, "Preestimation-based array interpolation approach to coherent source localization using multiple sparse subarrays," IEEE Signal Processing Letters, vol. 16, no. 2, pp. 81-84, 2009.

[24] Y. I. Abramovich, N. K. Spencer, and A. Y. Gorokhov, "Positivedefinite Toeplitz completion in DOA estimation for nonuniform linear antenna arrays. II. Partially augmentable arrays," IEEE Transactions on Signal Processing, vol. 47, no. 6, pp. 15021521, 1999.

[25] F.-M. Han and X.-D. Zhang, "An ESPRIT-like algorithm for coherent DOA estimation," IEEE Antennas and Wireless Propagation Letters, vol. 4, no. 1, pp. 443-446, 2005.

[26] J. Wang, S. Kwon, P. Li, and B. Shim, "Recovery of sparse signals via generalized orthogonal matching pursuit: a new analysis," IEEE Transactions on Signal Processing, vol. 64, no. 4, pp. 10761089, 2016.

[27] S. K. Sahoo and A. Makur, "Signal recovery from random measurements via extended orthogonal matching pursuit," IEEE Transactions on Signal Processing, vol. 63, no. 10, pp. 25722581, 2015.

[28] E. BouDaher, F. Ahmad, and M. G. Amin, "Sparse reconstruction for direction-of-arrival estimation using multi-frequency co-prime arrays," EURASIP Journal on Advances in Signal Processing, vol. 2014, article no. 168, 2014.

[29] Q. Shen, W. Liu, W. Cui, S. Wu, Y. D. Zhang, and M. G. Amin, "Low-complexity direction-of-arrival estimation based on wideband co-prime arrays," IEEE Transactions on Audio, Speech and Language Processing, vol. 23, no. 9, pp. 1445-1456, 2015.

[30] Y. Chi, L. L. Scharf, A. Pezeshki, and A. R. Calderbank, "Sensitivity to basis mismatch in compressed sensing," IEEE Transactions on Signal Processing, vol. 59, no. 5, pp. 2182-2195, 2011.

[31] C. El Kassis, J. Picheral, G. Fleury, and C. Mokbel, "Direction of arrival estimation using EM-ESPRIT with nonuniform arrays," Circuits, Systems, and Signal Processing, vol. 31, no. 5, pp. 17871807, 2012.

[32] D. Malioutov, M. Cetin, and A. S. Willsky, "A sparse signal reconstruction perspective for source localization with sensor arrays," IEEE Transactions on Signal Processing, vol. 53, no. 8, pp. 3010-3022, 2005. 


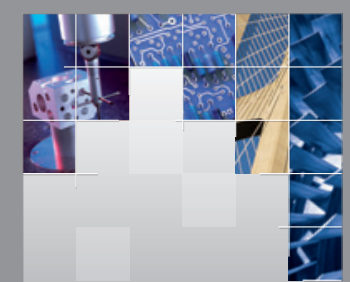

\section{Enfincering}
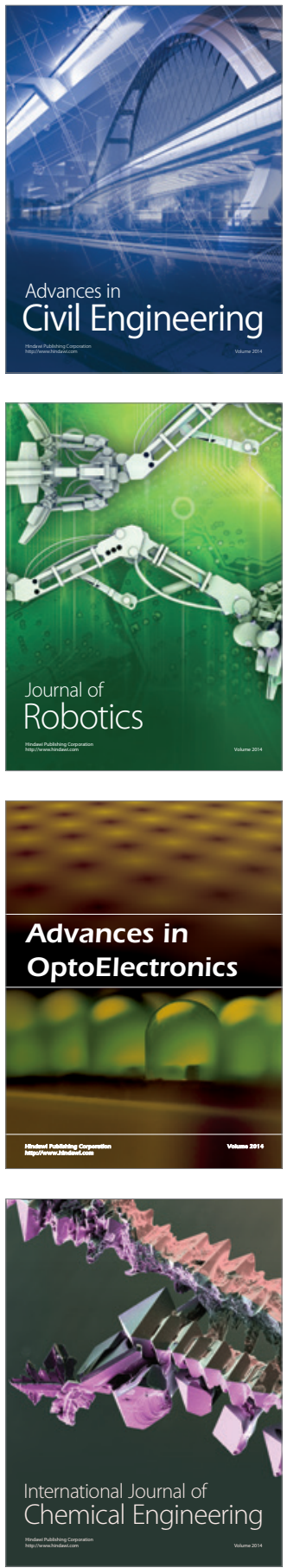

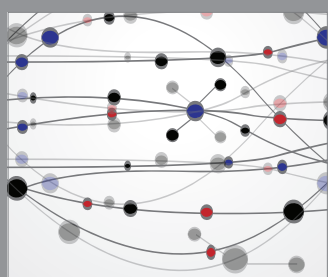

The Scientific World Journal

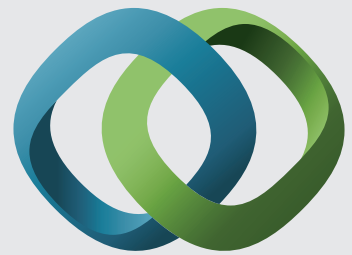

\section{Hindawi}

Submit your manuscripts at

https://www.hindawi.com
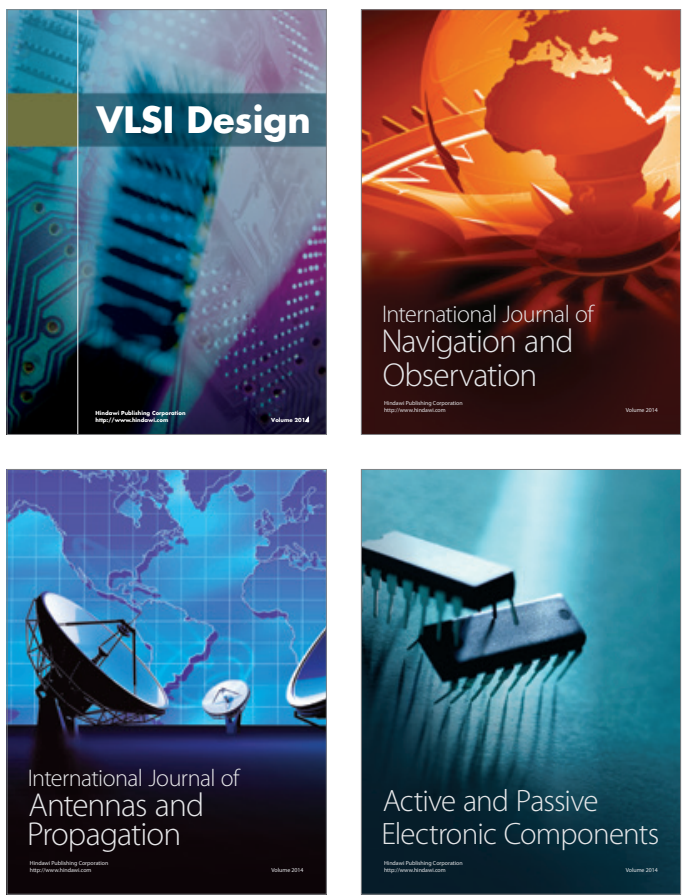
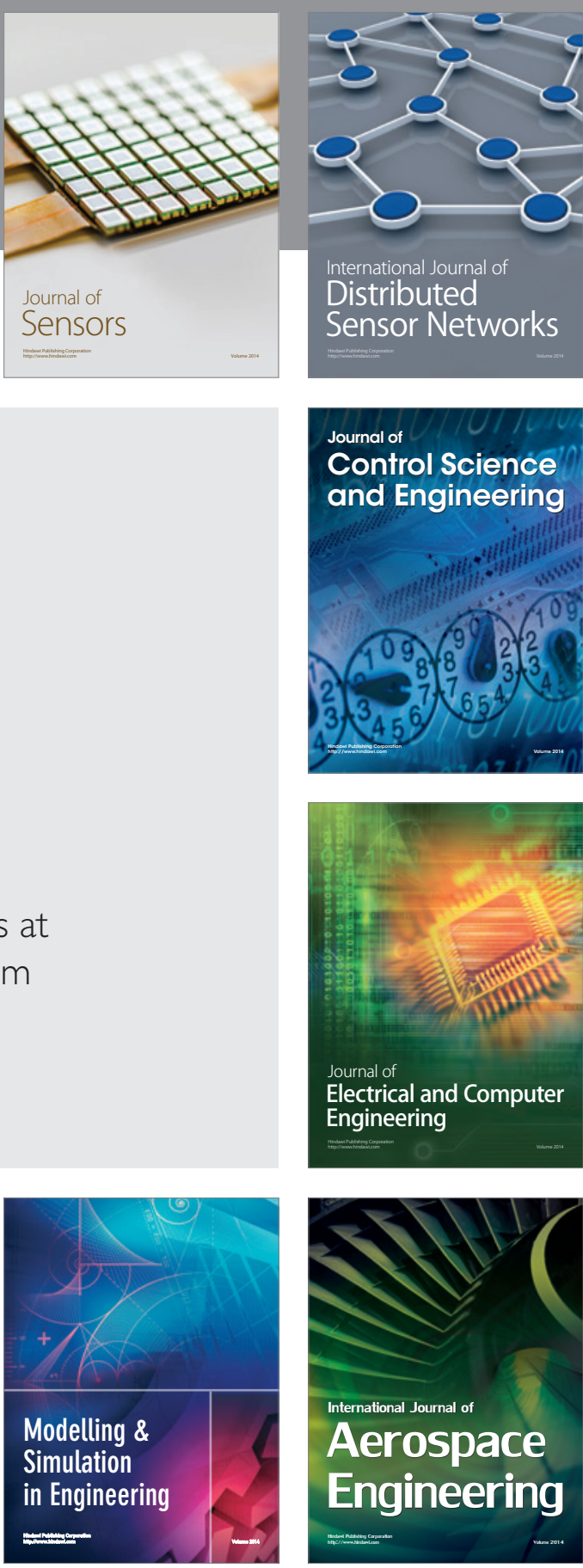

International Journal of

Distributed

Sensor Networks

$-$

Joumal of

Control Science

and Engineering
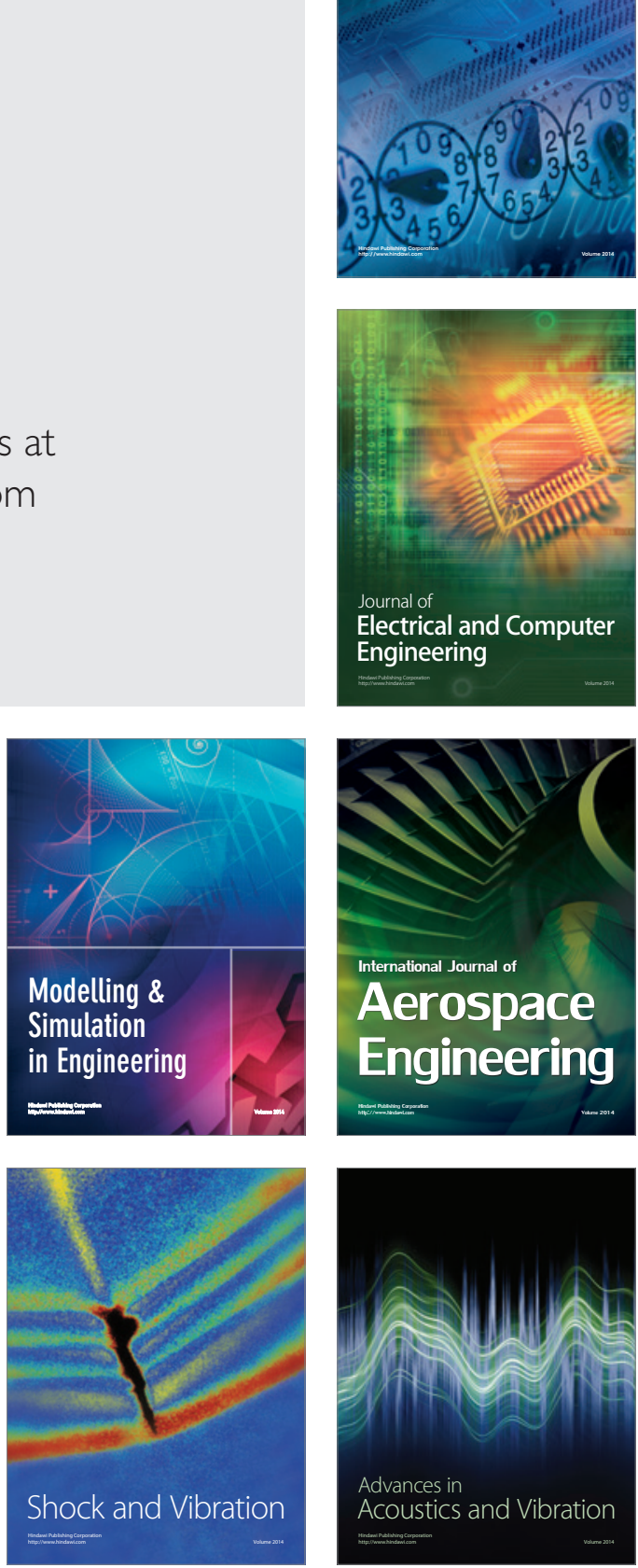